\title{
L'impartialité au cœur de l'autorité du juge Approches philosophiques
}

\author{
Julie Allard
}

\begin{abstract}
Résumé: L'autorité du juge est souvent menacée par la subjectivité des décisions rendues, auquel on oppose un impératif d'impartialité. Du point de vue philosophique, l'impartialité, liée à sa position tierce du juge, est perçue comme sa capacité à mettre entre parenthèse sa subjectivité, ses opinions et convictions personnelles, ses sentiments et ses affects, qui pourraient influencer de manière arbitraire sa décision. Cette impartialité est conçue par la tradition rationaliste comme une distance avec le corps et ses fragilités : il s'agit de juger en pur esprit (Platon) ou en être inanimé (Montesquieu). Deux traditions contestent cette conception de l'impartialité : selon les empiristes, aucun jugement n'est possible en surplomb, sans affect ni émotion (Hume); selon les sceptiques, la prétendue impartialité du juge est un rideau de fumée qui cache le pouvoir des juges et surtout, l'arbitraire de leurs décisions (Montaigne). Les penseurs contemporains comme Hannah Arendt tentent de concilier l'impératif de rationalité et le recours aux sentiments et à l'imagination pour bien juger, et aboutissent ainsi à une notion d'impartialité qui n'est pas ontologique, mais conçue comme un retour critique sur soi.
\end{abstract}

Mots-clefs : impartialité, rationalisme, empirisme, scepticisme, Arendt, acte de juger.

Julie Allard: Julie Allard est Doyenne de la Faculté de droit et de criminologie de l'Université libre de Bruxelles, où elle enseigne la philosophie du droit, et chercheure associée à l'Institut des Hautes sur la Justice. Elle participe depuis 2004 à la formation au sein de l'Ecole Nationale de la Magistrature. Ses recherches portent sur la figure du juge et son action.

Il existe au moins trois sources possibles d'autorité pour le juge. L'autorité du juge peut tout d'abord reposer sur sa capacité à prendre les bonnes décisions dans chaque situation. Ce sont ses qualités personnelles qui donnent ainsi au juge de l'autorité : charisme, réputation, mais aussi sagesse et prudence. C'est ce que pointe la notion de vertu chez Aristote $^{1}$. Ensuite, le juge peut également tenir son autorité du peuple, comme le veut par exemple la tradition de common law aux Etats-Unis. On juge en tant que citoyen et on s'adresse

\footnotetext{
${ }^{1}$ Chez Aristote, la vertu est une disposition qui doit aider les individus à s'orienter dans les affaires humaines, c'est-à-dire dans ce domaine où aucune certitude n'est possible et où, à défaut de savoir, nous avons la sagesse, qui s'acquière par expérience et permet de prendre les bonnes décisions dans une situation particulière. «On devient architecte en construisant; on devient musicien en faisant de la musique. Tout de même, on devient juste en pratiquant la justice, sage en cultivant la sagesse ; courageux en exerçant le courage » Aristote, Ethique à Nicomaque, Livre V, Chap. IV, Paris, Livre de Poche, 1992, p.78). Voir aussi : J. Allard, A. Garapon et F. Gros, Les vertus du juge. Une anthologie philosophique, Paris, Dalloz, 2007.
} 
à l'opinion quand on juge, d'où le langage naturel utilisé dans les décisions américaines. Enfin, l'autorité du juge peut renvoyer à une transcendance, à quelque chose qui le dépasse et dont il est le serviteur: Dieu, le Roi, l'Etat, la Loi. C'est cette option, choisie en France, qui consacre une dimension sacrée à la fonction de juge juge.

Quelle que soit la source retenue pour fonder l'autorité du juge, celle-ci est menacée par la subjectivité des décisions rendues. La finitude du juge, son éventuel manque de probité, comme la faillibilité de ses décisions, minent son autorité et la confiance que l'on porte aux décisions rendues. Cette subjectivité rend la justice potentiellement arbitraire, au sens où les décisions rendues relèveraient de la volonté personnelle du juge.

Cette difficulté est souvent désignée par le problème de la partialité et de l'impartialité du juge. Certes, du point de vue du droit positif, l'impartialité concerne seulement les relations du juge aux parties au procès ${ }^{2}$. Mais l'impartialité du juge renvoie aussi, au-delà du droit positif, à la position tierce du juge, qui fonde son autorité et sa légitimité pour arbitrer un différend. L'impartialité signifie en effet que le juge, avant que les parties aient plaidé en tout cas, n'a pas de préjugés ni de partis pris. Cela suppose donc qu’il est capable aussi de mettre entre parenthèse sa subjectivité, ses opinions, sentiments, convictions, qui pourraient influencer sa décision, et que cette capacité fonde l'autorité que l'on peut ou non reconnaitre à sa décision.

Pour évoquer plus en profondeur ce lien entre impartialité et autorité du juge, je propose de l'aborder en trois temps. Dans un premier temps, je reviendrai sur une tradition philosophique dominante, la tradition rationaliste, selon laquelle l'impartialité exige des juges une espèce de distance qui leur permettrait de juger rationnellement ou encore, comme dit Platon, de juger en purs esprits (I.). Dans un deuxième temps, je montrerai que cette première tradition est l'objet d'au moins deux critiques (II.). D'une part, la tradition empiriste conteste l'idée selon laquelle la justice peut se rendre sans les sentiments, sans les affects, sans les émotions. D'autre part, la tradition sceptique démontre le caractère mythologique de l'impartialité pensée par les rationalistes, qui n'est qu'un rideau de fumée cachant les vraies motivations des juges et leur pouvoir. Enfin, dans un troisième temps, une voie médiane pourra être empruntée à partir de la pensée de Hannah Arendt, qui tente de concilier impartialité et humanité, distance et sensibilité, en réinterprétant à nouveaux frais le terme même d'impartialité et l'exigence qu'elle incarne pour le juge (III.).

\section{L'impartialité dans la tradition rationaliste}

Selon une tradition philosophique relativement dominante, d'inspiration rationaliste, l'impartialité implique une mise à distance des sentiments, des opinions, des convictions, des émotions, afin de fonder le jugement en raison - étant entendu que c'est la raison qui sauve la subjectivité humaine de son arbitraire. La philosophie de Platon donne à voir un bel exemple de cette approche de l'acte de juger et de son impartialité.

\footnotetext{
${ }^{2}$ Les liens réprouvés par le droit entre le juge et les parties sont par exemple la parenté, l'alliance, l'amitié ou l'inimitié, les rapports d'intérêt ou de subordination. Les liens réprouvés par le droit entre le juge et les parties sont par exemple la parenté, l'alliance, l'amitié ou l'inimitié, les rapports d'intérêt ou de subordination. C'est d'ailleurs au nom de ce principe que les plaideurs ont le droit de récuser des juges suspects à leurs yeux de partialité (Article 297 du Code de procédure pénal et 341 et s. du Nouveau Code de procédure civile), c'est-àdire susceptibles d'être influencés, dans leur décision, par leur position à l'égard des parties.
} 
Platon a été hanté toute sa vie par le procès de son maitre Socrate et par l'injustice dont il a, selon lui, été victime. Alors que Socrate critique ouvertement les hommes forts du nouveau régime athénien ${ }^{3}$, il fait en effet l'objet d'un procès politique qui aboutit à sa condmanation à mort. Or, comme le veut le système judiciaire athénien, ce ne sont pas des juges professionnels qui ont jugé Socrate, mais des citoyens tirés au sort et réunis en assemblée (environ 500 dans le procès de Socrate). La critique de la justice humaine que Platon va en tirer sera donc aussi une critique de la justice populaire athénienne $e^{4}$

Dans les différents récits que Platon fait de ce procès, notamment dans l'Apologie de Socrate, il va, comme à son habitude, présenter les choses sous la forme d'une dualité : d'un côté, la figure du philosophe incarnée par Socrate, qui détient la vérité, de l'autre la figure des sophistes, ces habiles manipulateurs des apparences et du langage, qui se situent d'emblée du côté du pouvoir, pouvoir dont le procès n'est donc qu'un instrument ${ }^{5}$. C'est parce que la vérité de la pensée philosophique met en cause les apparences et donc le pouvoir qui s'appuie sur elles pour se maintenir que Socrate est jugé. Platon conclut donc du procès de Socrate que la justice (des hommes) est du côté du pouvoir et non de la vérité (contrairement à l'idée de justice). La condamnation de Socrate ne peut en effet avoir lieu qu'en vertu du divorce de la justice, comme idéal, et du droit, comme pratique concrète. Cette figure "négative » du juge présuppose donc aussi une figure « positive » du juge, un idéal par rapport auquel Platon dénonce la parodie de justice qu'illustre selon lui le procès de Socrate.

Plus exactement, Platon constate que la justice est rendue par des hommes qui se laissent trop influencer par des éléments qui ne devraient pas être pris en compte, notamment le langage utilisé par les professionnels du droit, ou encore les apparats dont se drappent certains justiciables. Seule la vérité, pense Platon, devrait compter dans un tribunal, et elle est cachée, masquée, par une séries de filtres qui, in fine, corrompent la justice.

Cette conception de la justice est nourrie de la métaphysique platonicienne, selon laquelle l'être des choses est masqué par des apparences trompeuses. Le corps et les sens, à travers lesquels nous appréhendons le monde, nous trompent au sujet de ce dernier. Pour voir le monde réel, tel qu'il est vraiment, il faut selon Platon l'appréhender non avec le corps mais avec l'esprit.

\footnotetext{
${ }^{3}$ Le procès de Socrate a lieu en 399 avant Jésus-Christ, à un moment de fragilité de la démocratie athénienne : Athènes sort à peine de la tyrannie imposée après la défaite contre Sparte par le régime des Trente, une oligarchie qui règne sur Athènes pendant quelques mois, semant la terreur, et dont le leader, Critias, est un ancien disciple de Socrate. Socrate lui-même, parce qu'il plaide pour fonder la cité sur le savoir vrai et non sur l'opinion majoritaire, n'est pas perçu comme un réel démocrate.

${ }^{4}$ De même, la procédure se veut démocratique et accessible à tous les citoyens : dans le cas de Socrate, une plainte est déposée par des citoyens - Mélitos, Anytos et Lycon -, qui l'accusent de corrompre la jeunesse, de réfuter l'existence des dieux de la cité et de chercher à introduire de nouvelles divinités. Une fois la plainte admise, ce sont ces mêmes citoyens qui vont porter l'accusation. Quant à Socrate, il assure seul sa défense, et ceci n'est pas un hasard au regard da sa philosophie : il n'a jamais fait confiance à l'art rhétorique des avocats, qui n'est selon lui nécessaire que quand on a quelque chose à se reprocher. Autrement dit, il refuse de préparer sa défense, arguant qu'il lui suffira de dire la vérité pour que justice éclate. Lors du vote, Socrate est condamné à mort à une courte majorité des voix et refuse l'exil qui lui est suggéré par ses disciples.

${ }^{5}$ Dans le récit qu'il fait de ce procès, notamment dans l'Apologie de Socrate, Platon dresse un portrait au vitriole du juge et de l'avocat, qui exercent un pouvoir qui ne repose que sur des apparences et dont la rhétorique est l'instrument. Le philosophe, incarné par la figure de Socrate, est l'homme libre qui n'a pas cédé aux séductions du pouvoir, qui ne s'est pas rendu esclave de la rhétorique, de cette argumentation creuse qui vide les idées de leur substance pour ne considérer que les apparences. "L'orateur n'est pas l'homme qui fait connaitre, aux tribunaux, ou à toute autre assemblée, ce qui est juste et ce qui est injuste ; en revanche, c'est l'homme qui fait croire que le juste, c'est ceci et l'injuste, c'est cela, rien de plus » (Platon, Gorgias, 454e-455a, Paris, GarnierFlammarion, 1993, p.141).
} 
Dans le Gorgias, Platon expose les conséquences de sa pensée sur le plan de la justice et la conception de l'impartialité qui en découle. C'est Socrate qui parle et raconte à Calliclès un mythe qui évoque la justice : «Du temps de Cronos et au commencement du règne de Zeus, c'étaient des vivants qui jugeaient d'autres vivants, et ils rendaient leur sentence le jour où ceux-ci devaient mourir. Or les jugements étaient mal rendus. [...] "Ie vais cesser ce mal, dit Zeus. Si les jugements jusqu'ici sont mal rendus, c'est qu'on juge les hommes encore vêtus, car on les juge de leur vivant. Or beaucoup d'hommes, ayant des âmes mauvaises, sont revêtus de beaux corps, de noblesse, de richesse [...]. Les juges sont alors frappés de stupeur devant cet appareil; en outre, comme ils siègent eux-même dans un appareil analogue, ayant devant l'âme des yeux, des oreilles, tout un corps qui les envloppe, tout cela fait obstacle, à la fois chez eux-mêmes et chez. ceux qu'ils ont à juger. [... Il faut qu'on les juge dépouillés de tout cet appareil [...]. Le juge aussi sera nu et mort, son âme voyant directement l'âme de chacun aussitôt après la mort [...]. Autrement, point de justice exacte $»^{6}$.

Ce mythe consacré à l'impartialité renvoie au problème de l'humanité des juges. Pour Platon, un bon juge, un juge impartial, doit se dépouiller de toutes ses particularités, de tous ses vêtements selon le symbole repris par Platon, qui font obstacle à la Justice. Il doit donc être nu et ceux qui comparaissent devant lui doivent également être nus, pour que les apparences ne distordent pas la réalité. Or «nus » chez Platon signifie plus spécifiquement « sans corps »: le bon magistrat juge seulement avec l'esprit, sans yeux et sans oreilles, dit Platon. Et bien entendu, ne peuvent être nus que les juges qui sont morts. Au fond, seul un mort peut faire preuve d'une telle impartialité, où il jugerait en pur esprit rationnel, sans être jamais ni affecté ni touché par ce dont il juge. Platon pense donc la vraie justice comme une justice désincarnée (sans corps).

Dans le même esprit, bien que le contexte de la modernité soit radicalement différent, Montesquieu propose l'image idéale du juge-machine. C'est le plus célèbre passage de L'esprit des lois : «Les juges de la Nation ne sont que la bouche qui prononce les paroles de la loi; des êtres inanimés qui n'en peuvent modérer ni la force ni la rigueur ${ }^{7}$. Le juge de Montesquieu est donc inanimé (il n'a pas d'âme), il n'est plus qu'une mécanique. Les juges, dans les termes de Montesquieu, sont dépersonnalisés : "On craint la magistrature et non les magistrats », car les juges ne décident pas en leur nom propre, mais au nom de la Loi. Comme tels, la subjectivité des juges, non seulement n'a pas sa place, mais doit être effacée, de sorte que les juges soient interchangeables entre eux et que la justice rendue soit la même quel que soit le juge qui la rend.

C'est ici le vieux rêve de la pensée mécaniciste qui est traduit dans les termes du droit : une justice automatique, dans laquelle les machines seraient les garantes du droit, serait plus impartiale et moins arbitraire que des normes appliquées par des humains, par définition imparfaits en raison de leurs limites cognitives, des influences qu'ils subissent, voire des corruptions dont ils font l'objet... Ainsi Napoléon, dans une séance du Conseil d'Etat le 7 mai 1806, déclarait-il pour critiquer une décision de justice relative au sort des juifs : «Les juges n'ont pas de pouvoir discrétionnaire; ce sont des machines physiques au moyen desquelles les lois sont exécutées comme l'heure est marquée par l'aiguille d'une montre $»^{8}$.

Que ce soit chez Platon ou chez Montesquieu, dont les idées perdurent dans la tradition républicaine française, les qualités humaines et subjectives du juge ne sont donc pas

\footnotetext{
${ }^{6}$ Platon, Gorgias, Paris, Les Belles Lettres, 2002, p.247.

${ }^{7}$ Montesquieu, De l'esprit des lois I, Livre XI, Chap. VI, Paris, Flammarion, 1979, p.301.

${ }^{8}$ Discours cité notamment dans : B. Philippe, Etre juif dans la société française. Du Moyen Âge à nos jours, Paris, Editions Complexe, 1999, p. 167.
} 
importantes, elles sont mêmes complètement évacuées, comme étant ce qui corrompt le jugement. L'impartialité attendue est pensée au contraire comme une dépersonnalisation, les juges devenant des êtres désincarnés, des morts ou des machines.

A sa façon, le développement de technologies numériques et de l'intelligence artificelle dans le droit poursuit le rêve de pouvoir remplacer l'homme et ses défaillances dans les processus de décision. En prenant la place de l'humain, les systèmes automatisés ne pourraient-ils pas en effet rendre une justice plus fiable, voire plus juste que lui ? Cette question repose implicitement sur l'idée selon laquelle l'humain est un être doué de raison mais aussi d'émotions, d'intérêts, de craintes, de préjugés, et donc, en tant que tel, un être partial et influençable. Les limites de son esprit, ses préjugés, ses opinions, ses croyances sont pointés du doigt comme les sources principales de ses erreurs de jugement et, en conséquence, des faiblesses de la justice. Tout au long de l'histoire des idées, influencée par le rationalisme, les philosophes ont donc porté un soupçon sur la justice humaine et critiqué l'impact qu'avait la personnalité du juge sur la justice rendue.

\section{Critiques de l'impartialité}

Deux courants philosophiques vont toutefois s'attaquer à cette représentation : l'empirisme, d'une part, et le scepticisme, d'autre part.

L'empirisme, tout d'abord, critique la position de surplomb qu'on assigne au juge et qui serait la condition de son impartialité. Si l'impartialité implique qu'on se situe «audessus de la mêlée », comment le juge peut-il comprendre et ensuite juger? Ne faut-il pas au contraire qu'il se mette au niveau du justiciable, qu'il partage les passions et les souffrances des victimes, qu'il accepte d'être touché, ému par ce qui doit être jugé ?

Les empiristes soutiennent sur ce point que l'acte de juger n'est pas un acte exclusivement rationnel. Il repose aussi sur les facultés sensibles, qui nous permettent d'éprouver des affects. Hume pense le problème sous la forme d'une articulation entre la raison et le sentiment dans l'acte de juger. Selon lui, les facultés rationnelles et sensibles agissent en deux temps. D'abord la raison permet de déterminer les faits et de suspendre le sentiment jusqu'à ce que ceux-ci soient établis. Mais, ensuite, seul le sentiment permet de juger, dit Hume. Sans l'intervention de la subjectivité et de ses affects, aucune évaluation, aucun jugement n'est possible.

Dans le Traité de la nature bumaine, Hume prend l'exemple du meurtre avec préméditation : on ne peut juger un tel acte avec la seule raison, car on y voit alors qu'un fait, qu'une information. C'est avec le cœur seul que le caractère criminel de l'acte apparaît. «Le vice vous échappe totalement tant que vous considérez l'objet. Vous ne pouvez jamais le trouver avant d'orienter la réflexion vers votre propre cœur et de constater qu'un sentiment de désapprobation s'élève en vous contre cet acte »?

Dans cette vision empiriste, on le voit, on admet une forme de partialité, faite d'affects notamment et sans laquelle aucun jugement n'est possible : c'est depuis notre position dans le monde que nous jugeons. Mais l'empirisme, nous le verrons dans un instant,

\footnotetext{
${ }^{9}$ D. Hume, Traité de la nature bumaine III (la morale), Paris, Flammarion, 1993, p.64. Il s'agit, chez Hume, d'une véritable théorie empiriste du jugement : condamner, par exemple, dit Hume, c'est « éprouver une impression ou un sentiment de blâme » (Idem).
} 
n'enferme pas le sujet dans sa subjectivité, car les sentiments peuvent aussi nous conduire à nous ouvrir aux autres et à leurs propres sentiments.

La seconde critique adressée à la vision rationaliste de l'impartialité est quant à elle d'inspiration sceptique. La pensée sceptique au sujet de l'impartialité des juges se nourrit d'un double constat. D'une part, la vision rationaliste qui pense l'impartialité comme la capacité à juger en pur esprit, dépouillé de toutes influences partiales, est un mythe : en réalité, nous sommes toujours pris dans une histoire singulière - un corps même - dont on ne peut se défaire - à moins d'être mort ou machine. D'autre part, l'empirisme ne fait que révéler la vraie nature de la justice mais n'en tire pas toutes les conclusions qui s'imposent. En réalité, la justice est totalement contingente et sensible aux humeurs des juges, à leur position sociale, à leur culture, etc. C'est un thème classique au XVIIème siècle, chez des auteurs comme Pascal ou Montaigne. Pascal, par exemple, souligne la relativité du droit et de la justice, qui varient au gré des mœurs (tout comme les sentiments). C'est le texte bien connu des Pensées : «Trois degrés d'élévation du pôle renversent toute la jurisprudence. Un méridien décide de la vérité, en peu d'années de possession les lois fondamentales changent. Le droit a ses époques, l'entrée de Saturne au Lion nous marque l'origine d'un tel crime. Plaisante justice qu'une rivière borne! Vérité au-deçà des Pyrénées, erreur au-delà $»^{10}$.

Montaigne, de son côté, dénonce le fait que le sort d'un homme tienne aux passions du juge, à une maladie, à une colère ou à un repas mal digéré. Dès lors que nous sommes des corps, dont les tourments perturbent notre jugement, mais aussi dès lors que nous nous rendons sensibles au monde, le jugement ne peut pas être droit. «Ce ne sont pas seulement les fiièvres, les breuvages et les grands accidents qui renversent notre jugement : les moindres choses du monde le bouleversent ... et par conséquent, à peine se peut-il rencontrer une seule heure en la vie où notre jugement se trouve en sa due assiette, notre corps étant sujet à tant de continuelles mutations, et étoffé de tant de cordes $»^{11}$.

Montaigne partage donc les prémisses du rationalisme de Platon : le corps et ses émotions perturbent inévitablement le jugement. Mais Montaigne va en revanche contester l'illusion des rationalistes à vouloir remédier à cette défaillance fondamentale du jugement par l'exercice de la raison. Les rationalistes, on l'a vu, entendent en effet limiter l'arbitraire humain en dépouillant le sujet jugeant de sa personnalité. Un auteur moderne comme Cesare Beccaria, par exemple, propose d'imposer à la décision une forme rationnelle, le syllogisme, qui va imposer la loi et empêcher que n'interviennent dans le jugement des considérations partiales, inévitables dès qu'on laisse le juge libre d'interpréter les lois. Reprenant ainsi l'hypothèse, évoquée par Montaigne, d'un jugement qui dépendrait de la digestion du juge, Beccaria affirme ainsi qu'il ne faut pas laisser libre cours à l'interprétation des lois, sans quoi «l'esprit d'une loi serait donc le résultat de la logique bonne ou mauvaise d'un juge, d'une digestion aisée ou pénible, de la faiblesse de l'accusé, de la violence des passions du magistrat, de ses relations avec l'offensé, enfin de toutes ces petites causes qui changent les apparences, et dénaturent les objets dans l'esprit inconstant de l'homme. Ainsi nous verrions le sort d'un citoyen changer de face, en passant à un autre tribunal, et la vie des malheureux serait à la merci d'un faux raisonnement, ou de la mauvaise humeur de son juge $»^{12}$.

L'espoir rationaliste est donc de briguer la partialité subjective des personnes par l'autorité impersonnelle de la loi, qui s'imposerait formellement et rationnellement. Pour des auteurs sceptiques comme Montaigne, il s'agit là au mieux d'une illusion rationaliste, au pire

\footnotetext{
${ }^{10}$ B. Pascal, Les pensées, t. 1, Ed. A. Molinier, Paris, 1877, p. 92.

${ }_{11}$ Montaigne, Essais, Paris, PUF, collection « Quadrige », 2004, II, 12, pp.564-565.

${ }^{12}$ C. Beccaria, Traité des délits et des peines, Paris, Editions Cujas, 1966, pp.69-70.
} 
d'une stratégie de dissimulation du pouvoir par la forme juridique et par la multiplicité des lois. Dans les Essais, Montaigne soutient en effet que jamais les lois n'ont permis de brider le pouvoir des juges et que, au contraire, la multiplication des lois renforce la licence avec laquelle les juges se permettent de décider.

Montaigne en déduit une critique acerbe de l'institution judiciaire, à laquelle il participa pourtant en tant que magistrat. Montaigne s'insurge notamment contre le langage délibérément abscons qui se joue souvent de l'ignorance et de l'incompréhension des justiciables et la constitution d'un corps spécifique de " gens de robe » avec ses intérêts propres, qui a tendance à devenir une corporation. En outre, selon Montaigne, l'attirail des lois, mais aussi l'institution avec ses procédures et ses rituels, avec sa rationalité, n'ont pas d'autre fonction que de donner à l'arbitraire des juges un vernis de légitimité. En ce sens, l'impartialité de la raison serait cette apparence que se donne celui qui veut donner libre cours à son arbitraire en toute impunité.

«Il est certain que les jugements se rencontrent parfois plus tendus à la condamnation, plus épineux et âpres, tantôt plus faciles, aisés et enclins à l'excuse [...] Au demeurant, cette maladie ne se découvre pas si aisément, si elle n'est du tout extrême et irrémédiable, d'autant que la raison va toujours, et tordue, et boiteuse, et déhanchée, et avec le mensonge comme avec la vérité. Par ainsi il est malaisé de découvrir son mécompte et dérèglement. J'appelle toujours raison cette apparence de discours que chacun forge en soi : cette raison, de la condition de laquelle il y en peut avoir cent contraires autour d'un même sujet, c'est un instrument de plomb et de cire, allongeable, ployable et accommodable à tous biais et à toutes mesures; il ne reste que la suffisance de le savoir contourner $»^{13}$.

Le scepticisme de Montaigne pourrait alors se résumer ainsi : non seulement la justice n'est pas impartiale, car elle est fondée sur des appréciations toutes subjectives des juges qui interprètent les lois à leur façon, mais elle dissimule cette dimension d'elle-même derrière une mécanique rationnelle, derrière un formalisme des règles et des procédures qui, en réalité, sert surtout leur pouvoir. Autrement dit: mieux vaut éviter d'avoir à faire à la justice et à ses « formules consacrées » qui déroulent des processus quasi mécaniques d'accusation, voire de condamnation anticipée, résumés dans l'image d'une " machine » dont les rouages s'enclenchent inexorablement, sans s'inquiéter outre mesure de ce qu'ils risquent de broyer.

Cette vision sceptique de la justice et de son impartialité se poursuit au fil des siècles pour s'incarner plus récemment dans le réalisme juridique américain. Ce dernier considère en effet les formes du droit comme des rideaux de fumée destinés à cacher la partialité constitutive de la position du juge : ce qui fait la décision d'un juge et, au-delà, ce qui fait le droit lui-même, ce ne sont pas des lois ou des procédures, c'est sa position dans la société, ses opinions, ses passions, et plus généralement tout ce qui le détermine dans sa singularité, voire sa trivialité. Beccaria, alors qu'il s’inquiétait de l'arbitraire, défendait un système où la décision rendue ne dépendrait pas de la digestion du juge. Les réalistes, au contraire, assument le fait que la décision du juge peut dépendre de ce qu’il a mangé au petit-déjeuner ${ }^{14}$ et affirment que le syllogisme judiciaire n'est là que pour nous le cacher.

\footnotetext{
${ }^{13}$ Montaigne, op. cit.

${ }^{14}$ L'affirmation, devenue une expression aux Etats-Unis (Justice is what judges ate at breakfast), est attribuée de manière contestée au juge Jerome Frank, figure du réalisme juridique américain, qui témoignait ainsi à l'aube des années $50:$ : Out of my own experience as a trial lanyer, I can testify that a trial judge, because of overeating at lunch, may be somnolent in the afternoon court-session that he fails to hear an important item of testimony and so disregards it when deciding the case. (...) Dickens' lovers well remember Perker's advice to Pickwick: "A good, contented, well-breakfasted juryman, is a
} 


\section{Retour critique sur soi}

Comment tenir compte du caractère mythologique et fantasmatique de l'impartialité pensée par Montesquieu et Platon sans renoncer au principe de l'impartialité et verser dans le scepticisme à l'égard de l'acte de juger ? Les empiristes, les premiers, tracent la voie. Pour Hume en effet, l'intervention de l'affect et des émotions dans le jugement, ne l'enferme pas dans l'égoïsme froid du sujet mais, au contraire, ouvre le jugement au sort d'autrui. La capacité à s'émouvoir serait, pour les empiristes, une façon de ne pas cantonner le jugement à la subjectivité pure du sujet raisonnant. C'est la théorie du sentiment moral, énoncé notamment par Adam Smith : "Aussi égoïste que l'homme puisse être supposé, il y a évidemment certains principes dans sa nature qui le conduisent à s'intéresser à la fortune des autres et qui lui rendent nécessaire leur bonheur, quoi qu'il n'en retire rien d'autre que le plaisir de les voir heureux $»^{15}$.

Hume et les empiristes évoquent ainsi un sentiment de sympathie qui nous rend sensibles à l'autre et à son histoire et nous permet, à ce titre, de l'évaluer puis de la juger. Ce sentiment de sympathie ne doit pas être confondu avec une connivence avec les êtres que l'on apprécie. C'est au contraire, dit Hume, un sentiment d'humanité par lequel, dans nos jugements, on fait abstraction de la proximité première (soi, son frère, son voisin, son compatriote) pour éprouver de la sympathie pour l'autre, quel qu'il soit, en tant qu'homme. «Principe d'humanité, dit aussi Hume, en lequel tous les hommes, à quelque degré, se rencontrent $»^{16}$.

La vision empiriste de l'acte de juger, si elle admet un élargissement du point de vue subjectif grâce à la sensibilité et l'imagination, reste marquée par l'idée d'une forme de partialité inétivable de l'acte de juger, au sujet de la quelle la raison demeure impuissante. C'est la raison pour laquelle des auteurs contemporains, comme Hannah Arendt, qui reprennent cette idée d'élargissement du point de vue subjectif, tentent aussi de l'intégrer à une théorie compatible avec le rationalisme, théorie où la réflexion du sujet - et pas seulement ses émotions - jouerait un rôle essentiel.

Arendt se représente en effet l'impartialité comme une exigence de la pensée critique, et non comme un état qui, par définition, serait inatteignable par un homme fait de chair et de sang. Arendt va donc tenter de sortir l'impartialité de l'écueil de l'ontologie : pour atteindre l'impartialité, soutient Arendt, on n'attend pas de celui qui juge qu'il soit vraiment impartial - et donc ou mort, ou Dieu, ou machine. Arendt propose au contraire de situer l'impartialité requise pour bien juger sur le plan non plus de l'être, mais de l'apparaître. Dans ce cas, ce qui compte, ce n'est pas d'être à distance des parties, des préjuges, des émotions, mais de paraitre à distance.

Ce déplacement dans l'approche de l'impartialité se fait en deux temps. Tout d'abord, on peut ressentir des émotions ou éprouver des craintes, déterminées par notre situation dans le monde et le rôle qu'on y joue, et néanmoins prendre une certaine distance à leur égard. La distance, ici, a un caractère réflexif car le juge n'est pas privé de sentiments (ou de son corps comme chez Platon). Par contre, il est capable de regarder ses propres sentiments

capital thing to get hold of. Discontented or hungry jurymen, my dear sir, always find for the plaintiff” 》 (J. Frank, Courts on Trial. Myth and Reality in American Justice, Princeton University Press, 1973, p.162).

15 A. Smith, Théorie des sentiments moraux, Paris, PUF, 2014, p.21.

${ }^{16}$ D. Hume, Enquête sur les principes de la morale, Paris, Flammarion, 1991, p.188. 
et de les évaluer. L'impartialité sera ainsi définie comme une forme de retour critique sur $\operatorname{soi}^{17}$.

Ce retour critique, qui définit selon Arendt l'impartialité, consiste paradoxalement à prendre en compte le point de vue des autres. «On accède à l'impartialité en prenant en compte le point de vue des autres ; l'impartialité ne résulte pas d'une position supérieure qui, parce qu'elle se situe hors de la mêlée, trancherait la querelle ${ }^{18}$. Au contraire, l'impartialité, dit Arendt, c'est la pensée élargie.

Et elle définit la pensée élargie en citant Kant dans la Critique de la faculté de juger: on parvient à la pensée élargie « en comparant son jugement aux jugements des autres, qui sont en fait moins les jugements réels que les jugements possibles, en se mettant à la place de tout autre $»^{19}$. L'impartialité n'est donc plus présentée comme un point de vue de surplomb, hors de la mêlée : il s'agit autant de distance avec soi que de proximité avec les autres. Il s'agit, dit Arendt, de « rendre les autres présents » en se mettant à leur place, en regardant le monde de leur point de vue.

D'où le deuxième moment du raisonnement d'Arendt sur l'impartialité, qui lui permet de dépasser la notion empiriste de sympathie : si on prend de la distance avec soi en se donnant le point de vue des autres, on devient capable d'évaluer nos propres émotions, sentiments, préjugés, etc. On peut alors, dit Arendt, leur faire passer une espèce d'épreuve d'universalisation: tous mes sentiments ne sont pas bons du seul fait que je les ressens et qu'ils justifient la décision qui m'arrange, et d'ailleurs tous mes affects ne sont pas en tant que tels communicables. Cette " communicabilité », cette capacité à partager du subjectif, est selon Arendt une épreuve pour le jugement. La communicabilité est en effet une restriction qui contraint le jugement : tous les préjugés et émotions ne peuvent servir d'arguments et de motivations. Etre impartial revient donc à trier dans ce qui fait notre subjectivité, entre ce que l'on communique pour le partager et ce qui doit rester de l'ordre de l'intime.

Aussi, quand on dit des juges qu'ils ne doivent pas manifester leurs préjugés ou leurs sentiments, cela ne revient pas à leur demander de les cacher (ce qui nourrirait la position sceptique). Il s'agit seulement d'insister sur le fait qu'ils ne peuvent s'appuyer sur ces sentiments et préjugés pour rendre raison de leur décision. L'impartialité est donc pensée par Arendt comme un effort de la réflexion, et même de l'imagination, et non un état: être impartial, ce n'est pas être sans appartenance, désincarné, mais c'est être capable de ne pas manifester ses appartenances ${ }^{20}$.

Si l'impartialité pour un juge implique qu'il ne doit pas manifester ses intérêts ou ses partis-pris, cela ne signifie donc pas qu'il n'a pas d'intérêt ou de parti-pris - ce qui est

\footnotetext{
17 « Penser de manière critique ne s'applique pas seulement aux doctrines et aux concepts que l'on reçoit des autres, aux préjugés et aux traditions dont on hérite; c'est précisément en appliquant les normes critiques à sa propre pensée que l'on apprend l'art de la pensée critique » (H. Arendt, Juger. Sur la philosophie politique de Kant, Paris, Seuil, 1991, p.70).

18 H. Arendt, op.cit., p.71.

${ }^{19}$ Kant, Critique de la faculté de juger, in CEuvres philosophies, t. II, Paris, Gallimard, coll. de la Pléiade, 1984, $\$ 40$.

${ }^{20}$ On retrouve la même idée dans un vieil adage de la langue anglaise, cité dès 1970 par la Cour européenne des droits de l'homme dans l'arrêt Delcourt c. Belgique: «La justice n'a pas seulement à être rendue, mais elle doit aussi paraître être rendue » (Voir Cour européenne des droits de l'homme, arrêt Delcourt c/ Belgique (req. $\mathrm{n}^{\circ}$ 2689/65), 17/01/1970 : "Justice must not only be done; it must also be seen to be done »). La CEDH plaide d'ailleurs pour une véritable théorie de l'apparence, qui n'est pas seulement procédurale, mais manifeste une certaine conception de cette juridiction à l'égard de la scène judiciaire en général. Dans l'arrêt Didier c. France, en 2002, la Cour affirme par exemple qu'en matière d'impartialité du juge, même les apparences peuvent revêtir de l'importance ». La Cour dira également, dans d'autres arrêts, que l'impartialité consiste à ne manifester aucun parti pris.
} 
impossible : une telle exigence passerait inévitablement pour un mythe. Mais cela ne veut pas dire non plus qu'il faut les cacher, comme si le juge était un hypocrite. Pour Arendt, en effet, l'apparaitre n'est plus ce qui cache (comme chez Platon) mais ce qui montre, ce qui donne à voir, il s'agit donc, pour le juge de choisir ce qu'il donne à voir, faisant ainsi le tri, dans ses sentiments et ses émotions, entre ce qui mérite ou non de sortir de sa pure intériorité. Le mode de pensée élargie induit lui-même ce type de tri dans nos représentations.

La réflexion d'Arendt est bien sûr inspirée par le cas du procès Eichmann, dont elle fut témoin et qui lui inspira sa réflexion sur le jugement et son impartialité. Adolf Eichmann, haut-fonctionnaire de l'administration nazie, chargé d'organiser la logistique des trains de la déportation, est jugé en Israël dans les années 60. Arendt rend compte de ce procès pour un journal américain, et découvre un homme ordinaire qui prétend sans cesse avoir agi sous le troisième Reich en respectant la loi. " Autant qu'il pût en juger, Eichmann agissait, dans tout ce qu'il faisait, en bon citoyen respectueux de la loi. Il faisait son devoir, répéta-t-il à la police et au tribunal. [...] Et il finissait par insister [...] sur l'obéissance aveugle, - obéissance de cadavre comme il disait lui-même $»^{21}$.

Arendt explique cette attitude d'obéissance par l'incapacité d'Eichmann à émettre le moindre jugement : il appliquait la loi de son époque de façon aveugle - sans yeux et sans oreille pour parodier Platon. Le mythe du juge «bouche de la loi » était ainsi poussé à la caricature : l'application de la loi peut en effet, découvre Arendt, paralyser le jugement. C'est une « obéissance de cadavre », dit-elle, un cadavre qui n'est pas sans évoquer les juges morts de Platon, ou même les êtres inanimés de Montesquieu.

${ }^{21}$ H. Arendt, Eichmann à Jérusalem, Paris, Gallimard, 1966, p.152. 